\title{
灌浆期高温对小麦籽粒淀粉的积累、粒度分布及相关酶活性的影响
}

间素辉 尹燕枰 李文阳 梁太波 李 勇 邬云海王平 耿庆辉
戴忠民 王振林

(作物生物学国家重点实验室 / 山东农业大学农学院, 山东泰安 271018)

摘 要: 以不同耐热性品种济麦 20 和鲁麦 21 为材料, 于花后 5 9 d 进行高温处理, 研究了小麦灌浆期高温对籽粒淀 粉的积累、粒度分布及合成相关酶活性的影响。结果表明, 灌浆期高温显著降低籽粒淀粉积累量, 显著降低籽粒淀粉、 支链淀粉含量, 提高直链淀粉含量和直/支链淀粉比例。高温对济麦 20 籽粒淀粉积累的影响程度较鲁麦 21 大。灌浆 期高温使小麦籽粒 $\mathrm{A}$ 型淀粉粒的体积、数量和表面积百分比显著增加, B 型淀粉粒这 3 指标则显著降低。高温处理 后, 济麦 20 籽粒蔗糖合酶(SS)、ADPG 焦磷酸化酶(AGPP)、可溶性淀粉合酶(SSS)、束缚态淀粉合酶(GBSS)活性与 对照无显著差异, 而鲁麦 21 上述酶活性则高于对照。济麦 20 、鲁麦 21 籽粒上述酶活性分别于花后 $15 \mathrm{~d}$ 和 $20 \mathrm{~d}$ 开始 低于对照。与其他淀粉合成相关酶相比, 高温对籽粒 GBSS 活性的影响程度较小。两品种处理间籽粒蔗糖含量及 SS、 AGPP、SSS 和 GBSS 活性的变化趋势, 与其籽粒淀粉积累量的变化趋势基本一致。灌浆期高温使籽粒淀粉积累量降 低, 主要因高温抑制了籽粒灌浆中后期的淀粉合成, 这是由籽粒蔗糖供应不足和籽粒淀粉合成相关酶活性下降所造 成的。

关键词: 小麦; 高温; 淀粉; 淀粉粒; 酶活性

\section{Effect of High Temperature during Grain Filling on Starch Accumulation, Starch Granule Distribution, and Activities of Related Enzymes in Wheat Grains}

YAN Su-Hui, YIN Yan-Ping, LI Wen-Yang, LIANG Tai-Bo, LI Yong, WU Yun-Hai, WANG Ping, GENG Qing-Hui, DAI Zhong-Min, and WANG Zhen-Lin*

(National Key Laboratory of Crop Biology / Agronomy College, Shandong Agricultural University, Tai'an 271018, Shandong, China)

\begin{abstract}
In order to investigate the effects of high temperature at early grain filling stages (5-9 d after anthesis) on grain starch formation of wheat (Triticum aestivum L.), cultivars Jimai 20 (weak heat tolerance) and Lumai 21 (strong heat tolerance), were used with a heat stress treatment in the field by plastic sheds. Starch accumulation, starch granule distribution, and changes of enzymes activities involved in starch synthesis were examined. The results showed that heat stress after anthesis remarkably reduced starch accumulation at maturity. The total starch and amylopectin contents decreased significantly, but amylose content increased in heat treatments compared to control. Accordingly the ratio of amylose to amylopectin of heat treantment was significantly higher than that of control. The effect of high temperature on starch accumulation in Jimai 20 was greater than that in Lumai 21. The volume, number, and surface area percentage of A type starch granule were enhanced significantly, but those of B type starch granule reduced greatly under high temperature. After $5 \mathrm{~d}$ heating stress, the sucrose content, the activities of sucrose synthase (SS), adnosine diphosphate glucose pyrophosphorylase (AGPP), soluble starch synthase (SSS), and granule-bounded starch synthase (GBSS) increased in heat-treated grains of Lumai 21, but changed slightly in treated grains of Jimai 20 with no significant difference to control. These indexes of Jimai 20 and Lumai 21 became lower than those of control at 15 and $20 \mathrm{~d}$ post anthesis ( 6 and $11 \mathrm{~d}$ after heat stress removal), respectively. The GBSS activity was affected slightly by heat treatment. The results
\end{abstract}

基金项目: 国家自然科学基金项目(30270781，30571099); 教育部长江学者与创新团队发展计划项目(IRT0635); 山东省自然科学基金项目 (Y2005D13)

作者简介: 问素辉(1979-), 女, 山东菏泽人, 博士研究生, 研究方向为小麦品质生理与高产优质栽培技术。

“通讯作者(Corresponding author): 王振林。Tel: 0538-8241359; E-mail: zlwang@sdau.edu.cn

Received(收稿日期): 2007-10-08; Accepted(接受日期): 2007-12-20. 
indicate that the changes in grain sucrose content and the activities of SS, AGPP, SSS, and GBSS are companied with the changes in grain starch accumulation, and high temperature restrains starch synthesis at middle and late grain filling stages, which is caused by inferior sucrose providing capacity and low activities of enzymes (SS, AGPP, SSS, and GBSS) involved in starch synthesis.

Keywords: Winter wheat (Triticum aestivum L.); High temperature; Starch; Starch granule; Enzyme activity

淀粉组分构成对小麦面粉及其制品的品质具有重要 影响 ${ }^{[1]}$ 。小麦籽粒淀粉以淀粉粒的形式存在,一般分为体 积较大的 $\mathrm{A}$ 型和体积较小的 $\mathrm{B}$ 型淀粉粒 ${ }^{[2]}$ 。籽粒淀粉组分 及淀粉粒度分布因籽粒发育的不同阶段而不同 ${ }^{[2-5]}$ 。 Bechtel等 ${ }^{[5]}$ 研究提出小麦籽粒胚乳中 A型淀粉粒于花后 4 5 d 开始形成, B型淀粉粒则开始于花后 12 14 d。表明小 麦籽粒 $\mathrm{A}$ 型与 $\mathrm{B}$ 型淀粉粒的形成是一个受发育所调节的过 程。在小麦籽粒发育过程中, 温度等环境条件的变化不仅 影响小麦籽粒淀粉的形成与积累, 而且对籽粒淀粉组分 构成和淀粉粒度分布状态亦应具有调节效应, 然而关于 这方面的研究，国内未见报道。

小麦籽粒灌浆阶段的适宜温度为 $20 \sim 24^{\circ} \mathrm{C}$ 。籽粒灌浆 对高温的反应十分敏感 ${ }^{[6]}$ 。在我国及世界其他一些小麦种 植地区, 小麦籽粒发育过程中, 时常出现日均气温超过 $30^{\circ} \mathrm{C}$ 的短暂高温天气, 这对小麦籽粒产量和品质的形成 产生了极为不利的影响 ${ }^{[7-8]}$ 。高温对小麦籽粒淀粉合成相关 酶的活性有显著影响 ${ }^{[5,9-10]}$, 进而影响到籽粒淀粉的积累。 不同小麦品种对高温胁迫的反应存在显著差异。AI-Khatib 和Paulsen ${ }^{[11]}$ 研究表明, 在相同高温条件下, 耐热性强的小 麦品种籽粒产量受温度影响较小, 而热敏感品种籽粒产量 下降 $40 \%$ 。但是关于灌浆期短暂高温对不同耐热性小麦品 种籽粒淀粉形成影响的比较研究，迄今报道尚少。
为此, 本试验选用耐热性不同的两个小麦品种, 研究 籽粒灌浆期短暂高温对籽粒淀粉的积累、粒度分布及相关 酶活性的影响, 探讨高温影响淀粉形成的生理生化机制, 旨在为通过栽培措施改善籽粒淀粉品质提供理论依据。

\section{1 材料与方法}

\section{1 试验材料与设计}

试验于 2004 年 10 月一 2006 年 6 月在山东农业大学农 场进行。供试小麦品种为耐热性较弱的济麦 20 和耐热性较 强的鲁麦 21 。种植密度 180 万株 $\mathrm{hm}^{-2}$ 。小区面积为 $3 \mathrm{~m} \times 3$ $\mathrm{m}=9 \mathrm{~m}^{2}$ 。播种期为 2004 年 10 月 6 日和 2005 年 10 月 15 日。收获期为 2005 年 6 月 6 日和 2006 年 6 月 8 日。播种 前施入基肥纯氮 $120 \mathrm{~kg} \mathrm{hm}^{-2} 、 \mathrm{P}_{2} \mathrm{O}_{5} 75 \mathrm{~kg} \mathrm{hm}^{-2} 、 \mathrm{~K}_{2} \mathrm{O} 120 \mathrm{~kg}$ $\mathrm{hm}^{-2}$, 拔节期追施纯氮 $120 \mathrm{~kg} \mathrm{hm}^{-2}$ 。两年的试验结果趋势 一致, 本文采用 2005-2006 年试验数据进行分析。

参考Xu等 ${ }^{[12]}$ 的方法进行高温处理。增温棚用 $0.1 \mathrm{~mm}$ 厚无色透明聚乙烯塑料薄膜(透光率为 $90 \%$ ) 做成, 面积与 小区面积相同，高约 $1.5 \mathrm{~m}$, 增温棚两侧设 4 个通风口，以 不影响群体内通风状况。于花后 5 9 d, 每日 8:00-18:00 用增温棚进行高温处理 $(\mathrm{HT})$, 以不增温为对照 $(\mathrm{CK})$ 。表 1 为花后 5 9 d 处理间的温度日变化。

自小麦开花后 $10 \mathrm{~d}$ 起，每 $5 \mathrm{~d}$ 取样一次。部分置液氮

表 1 高温处理时增温棚内外温度日变化

Table 1 Diurnal changes of temperature inside and outside the sheds

\begin{tabular}{|c|c|c|c|c|c|c|c|c|c|}
\hline \multirow{2}{*}{$\begin{array}{c}\text { 处理 } \\
\text { Treatment }\end{array}$} & \multicolumn{9}{|c|}{ 时间 Time } \\
\hline & 9:00 & $10: 00$ & 11:00 & $12: 00$ & 13:00 & $14: 00$ & $15: 00$ & $16: 00$ & $17: 00$ \\
\hline $\mathrm{CK}$ & 22.6 & 25.2 & 27.3 & 29.4 & 31.7 & 31.4 & 28.5 & 26.5 & 24.9 \\
\hline HT & 27.5 & 34.8 & 38.9 & 39.2 & 39.8 & 39.4 & 39.0 & 36.2 & 29.3 \\
\hline
\end{tabular}

数据为花后 $5 \sim 9 \mathrm{~d}$ 温度日变化的平均值。CK、 $\mathrm{HT}$ (高温处理) 分别为棚外、内温度。

Data are the mean of the 5th to 9th day after anthesis. Temperatures of CK and HT (heat treatment) were examined outside and inside the sheds.

中速冻 $10 \mathrm{~min}$, 保存于 $-40^{\circ} \mathrm{C}$ 冰箱, 用于酶活性测定; 部 分置 $70^{\circ} \mathrm{C}$ 烘箱烘至恒重, 用于籽粒熫糖、淀粉含量测定及 淀粉粒径分析。

\section{2 测定项目与方法}

采用双波长比色法 ${ }^{[13]}$ 测淀粉含量。采用蒽酮比色法 ${ }^{[13]}$ 测蔗糖含量。参考Peng等 ${ }^{[4]}$ 的方法提取淀粉粒，用美国 贝克曼库尔特公司LS13320 激光衍射粒度分析仪分析淀 粉粒粒径分布。参照Douglas等 ${ }^{[14]}$ 的方法测蔗糖合酶 $(\mathrm{SS})$ 和腺 苷二磷酸葡萄糖焦磷酸化酶 (AGPP) 活性。参照 Nakamura等 ${ }^{[15]}$ 的方法测可溶性淀粉合酶 (SSS) 和束缚态 淀粉合酶(GBSS)活性。

\section{2 结果与分析}

\section{1 籽粒淀粉积累量}

高温处理后, 籽粒淀粉积累量均高于对照, 但品种 间存在差异, 济麦 20 籽粒淀粉积累量的升幅较鲁麦 21 小, 且于花后 $15 \mathrm{~d}$ 即较对照下降; 而鲁麦 21 籽粒淀粉积累量 较对照上升幅度高于济麦 20 , 且于花后 $20 \mathrm{~d}$ 才开始低于对 照(图 1)。花后 $35 \mathrm{~d}$ (成熟期), 两品种高温处理籽粒淀粉积累 量分别较对照下降 18.3\%(济麦 20)和 10.2\%(鲁麦 21)。说明 高温不利于小麦籽粒淀粉的积累; 且两品种对灌浆期高温 的耐受能力存在差异, 鲁麦 21 的耐高温能力较济麦 20 强。 


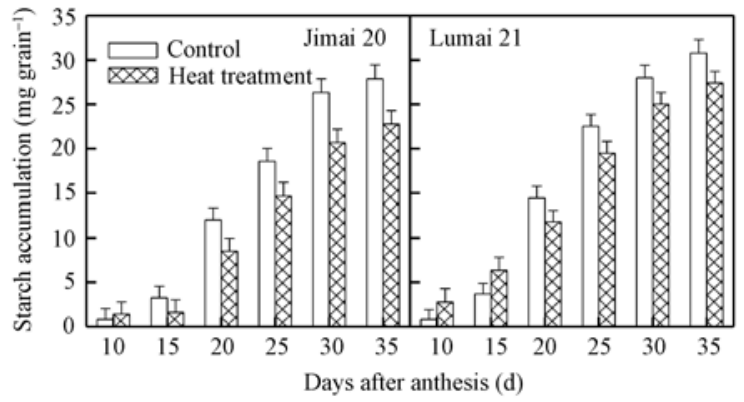

图 1 高温对小麦籽粒淀粉积累量的影响

Fig. 1 Effect of high temperature after anthesis on total starch accumulation in wheat grain

\section{2 籽粒淀粉含量}

小麦高温处理籽粒淀粉、支链淀粉含量极显著低于对 照, 直链淀粉含量、直/支比显著高于对照(表 2)。品种间比较, 济麦 20 高温处理淀粉及支链淀粉含量降幅较鲁麦 21 大。

2.3 淀粉粒度分布

小麦籽粒淀粉粒主要由 $<43 \mu \mathrm{m}$ 淀粉粒构成。与对照 相比, 高温显著降低 B 型 $(<10 \mu \mathrm{m})$ 淀粉粒体积、数量和表 面积百分比, 增加籽粒 $\mathrm{A}$ 型 $(10 \sim 43 \mu \mathrm{m})$ 淀粉粒体积、数量 和表面积百分比(表 3)。品种间比较，高温对耐热性较弱的 小麦品种济麦 20 籽粒 B 型淀粉粒降低幅度大于耐热性较 强的小麦品种鲁麦 21 。

表 2 高温对小麦成熟期籽粒淀粉含量的影响

Table 2 Effect of high temperature after anthesis on content of starch in mature grain

\begin{tabular}{cccccc}
\hline $\begin{array}{c}\text { 品种 } \\
\text { Cultivar }\end{array}$ & $\begin{array}{c}\text { 处理 } \\
\text { Treatment }\end{array}$ & $\begin{array}{c}\text { 直链淀粉含量 } \\
\text { Amylose content (\%) }\end{array}$ & $\begin{array}{c}\text { 支链淀粉含量 } \\
\text { Amylopectin content (\%) }\end{array}$ & $\begin{array}{c}\text { 淀粉含量 } \\
\text { Starch content (\%) }\end{array}$ & $\begin{array}{c}\text { 直/支比 } \\
\text { Ratio of amylose to amylopectin }\end{array}$ \\
\hline 济麦 20 & CK & $15.05 \pm 0.26 \mathrm{~b}$ & $48.69 \pm 0.43 \mathrm{a}$ & $63.74 \pm 0.68 \mathrm{a}$ & $0.309 \pm 0.003 \mathrm{c}$ \\
Jimai 20 & HT & $16.04 \pm 0.28 \mathrm{a}$ & $43.68 \pm 0.45 \mathrm{~b}$ & $59.72 \pm 0.72 \mathrm{~b}$ & $0.367 \pm 0.003 \mathrm{~b}$ \\
鲁麦 21 & CK & $18.16 \pm 0.34 \mathrm{~b}$ & $50.05 \pm 0.48 \mathrm{a}$ & $68.21 \pm 0.82 \mathrm{a}$ & $0.363 \pm 0.003 \mathrm{c}$ \\
Lumai 21 & HT & $19.11 \pm 0.37 \mathrm{a}$ & $47.62 \pm 0.39 \mathrm{~b}$ & $66.73 \pm 0.76 \mathrm{~b}$ & $0.401 \pm 0.004 \mathrm{~b}$ \\
\hline
\end{tabular}

表中数据为平均值标准差。同一品种内, 标以不同小写字母的值在处理与对照间差异显著。

Data in the table are mean $\pm S D$. For each cultivar, means followed by a different letter are significantly different between control (CK) and heat treatment (HT) at $P<0.05$.

表 3 高温对小麦成熟期籽粒淀粉粒度分布的影响

Table 3 Effect of high temperature after anthesis on starch granules distribution in mature grain

\begin{tabular}{|c|c|c|c|c|c|c|c|}
\hline \multirow{2}{*}{$\begin{array}{c}\text { 品种 } \\
\text { Cultivar }\end{array}$} & \multirow{2}{*}{$\begin{array}{c}\text { 处理 } \\
\text { Treatment }\end{array}$} & \multicolumn{2}{|c|}{ 体积 Volume (\%) } & \multicolumn{2}{|c|}{ 数目 Number (\%) } & \multicolumn{2}{|c|}{ 表面积 Surface area (\%) } \\
\hline & & $<10 \mu \mathrm{m}$ & $10 \sim 43 \mu \mathrm{m}$ & $<10 \mu \mathrm{m}$ & $10 \sim 43 \mu \mathrm{m}$ & $<10 \mu \mathrm{m}$ & $10 \sim 43 \mu \mathrm{m}$ \\
\hline 济麦 20 & CK & $41.8 \pm 0.4 \mathrm{a}$ & $57.9 \pm 0.3 \mathrm{~b}$ & $99.91 \pm 0.03 \mathrm{a}$ & $0.09 \pm 0.02 \mathrm{~b}$ & $82.6 \pm 0.5 \mathrm{a}$ & $17.4 \pm 0.3 \mathrm{~b}$ \\
\hline Jimai 20 & HT & $27.2 \pm 0.3 \mathrm{~b}$ & $72.2 \pm 0.4 \mathrm{a}$ & $99.62 \pm 0.04 \mathrm{~b}$ & $0.38 \pm 0.03 \mathrm{a}$ & $74.6 \pm 0.4 \mathrm{~b}$ & $25.1 \pm 0.2 \mathrm{a}$ \\
\hline 鲁麦 21 & CK & $38.7 \pm 0.3 \mathrm{a}$ & $60.9 \pm 0.3 \mathrm{~b}$ & $99.77 \pm 0.04 \mathrm{a}$ & $0.23 \pm 0.03 \mathrm{~b}$ & $75.9 \pm 0.5 \mathrm{a}$ & $24.0 \pm 0.3 \mathrm{~b}$ \\
\hline Lumai 21 & HT & $24.9 \pm 0.2 \mathrm{~b}$ & $74.2 \pm 0.4 \mathrm{a}$ & $99.51 \pm 0.03 \mathrm{~b}$ & $0.48 \pm 0.04 \mathrm{a}$ & $68.7 \pm 0.4 \mathrm{~b}$ & $30.9 \pm 0.4 \mathrm{a}$ \\
\hline
\end{tabular}

表中数据为平均值 \pm 标准差。同一品种内, 标以不同小写字母的值在处理与对照间差异显著。

Data in the table are mean $\pm S D$. For each cultivar, means followed by a different letter are significantly different between control (CK) and heat treatment (HT) at $P<0.05$.

\section{4 籽粒 SS 活性和蔗糖含量}

2.4.1 SS 活性由图 2 可以看出, 不同小麦品种籽粒 SS 活性对高温的耐受能力存在显著差异。5 9 d 高温处理 后, 耐热性较弱的小麦品种济麦 20 籽粒 SS 活性略高于对 照, 耐热性较强的小麦品种鲁麦 21 籽粒 SS 活性明显升 高。济麦 20 于花后 $15 \mathrm{~d}$ 即显著低于对照, 而鲁麦 21 则于 花后 $20 \mathrm{~d}$ 之后才表现出较对照下降的趋势。

2.4 .2 蔗糖含量高温胁迫后, 两小麦籽粒蔗糖含量 均高于对照, 鲁麦 21 上升幅度更为明显(图 2)。胁迫解除 后, 济麦 20 于花后 $15 \mathrm{~d}$ 籽粒蔗糖含量即较对照显著下降, 而鲁麦 21 则于花后 $20 \mathrm{~d}$ 低于对照。结合籽粒 SS 活性的 变化, 说明灌浆前期高温抑制了籽粒灌浆中后期淀粉合 成原料蔗糖向籽粒的供应。
2.5 籽粒淀粉合成相关酶活性变化

2.5.1 AGPP 活性 高温处理后, 鲁麦 21 籽粒 AGPP 活性较对照高, 济麦 20 籽粒 AGPP 活性与对照无显著 差异(图 3)。可见一定程度的热激能短期提高鲁麦 21 籽粒 AGPP 活性, 说明它在高温条件下具有较强的自动调节能 力。胁迫解除后, 济麦 20 、鲁麦 21 分别于花后 $15 \mathrm{~d}$ 和 20 $\mathrm{d}$ 开始低于对照。说明耐热性不同的小麦品种籽粒 AGPP 活性对高温的反应不同。

2.5.2 SSS 活性 由图 4 可以看出, 高温处理 $5 \mathrm{~d}$ 后, 两品种籽粒 SSS 活性均较对照高。高温解除后, 济麦 20 籽粒 SSS 活性于花后 $15 \mathrm{~d}$ 就开始低于对照, 鲁麦 21 则表 现较滞后, 于花后 $20 \mathrm{~d}$ 低于对照; 济麦 20 籽粒 SSS 活性 下降幅度显著大于鲁麦 21 。说明灌浆期高温条件下, 籽粒 支链淀粉的合成均受到抑制, 耐热性较强的小麦品种籽 
粒支链淀粉受抑程度小于耐热性较弱的小麦品种。

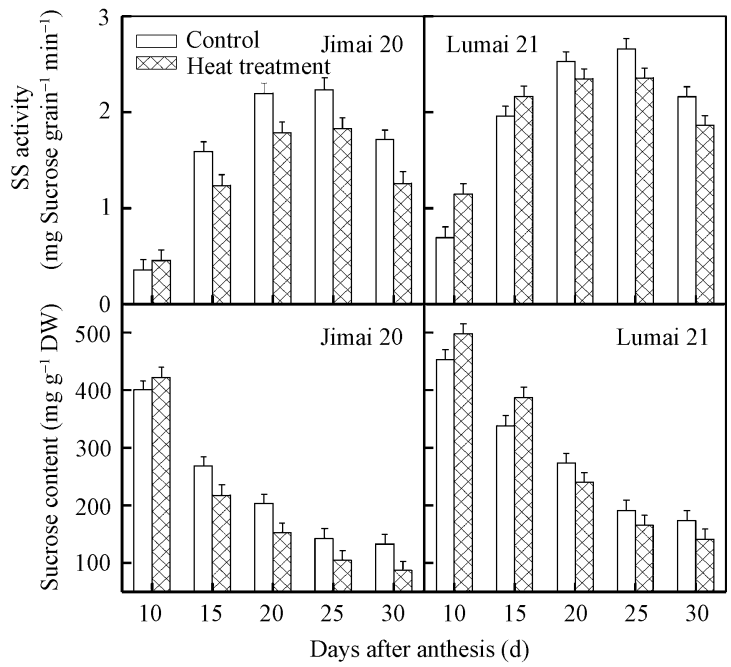

图 2 高温对小麦籽粒 SS 活性和蔗糖含量的影响

Fig. 2 Effect of high temperature after anthesis on SS activity and sucrose content in wheat grain

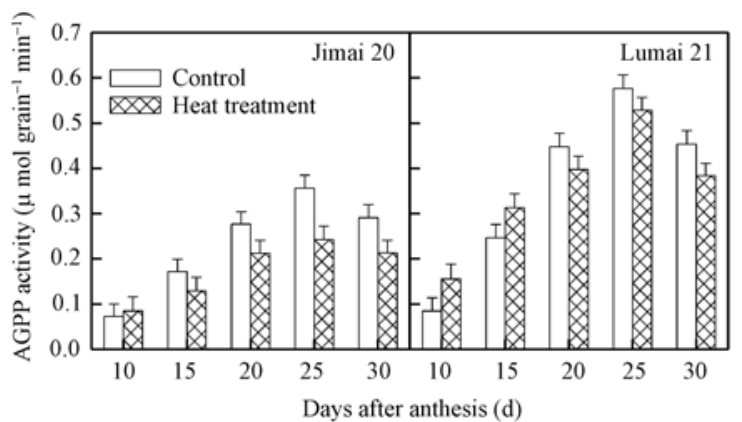

图 3 高温对小麦籽粒 AGPP 活性的影响

Fig. 3 Effect of high temperature after anthesis on the activity of AGPP in wheat grain

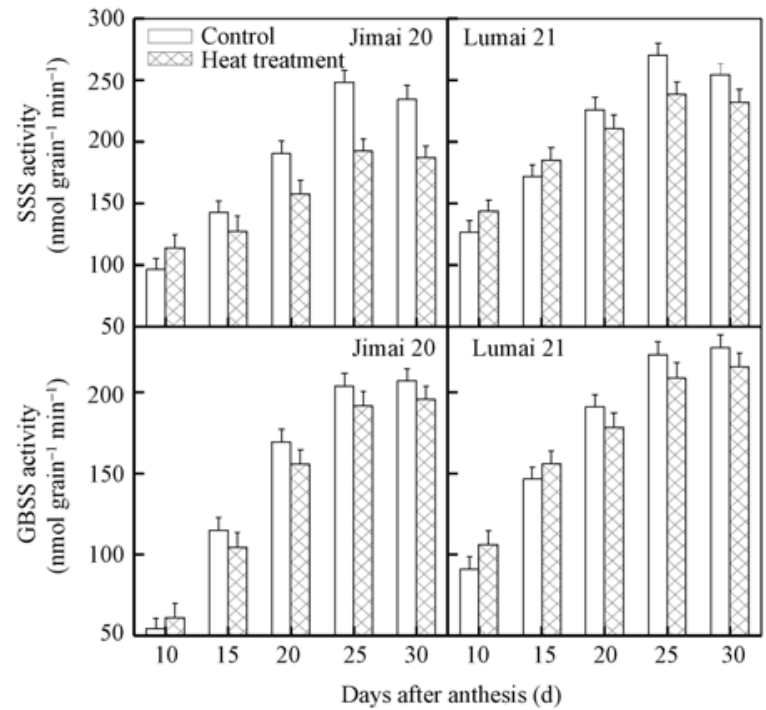

图 4 高温对小麦籽粒 SSS 和 GBSS 活性的影响

Fig. 4 Effect of high temperature after anthesis on activities of SSS and GBSS in wheat grain
2.5.3 GBSS 活性 图 4 显示, 高温处理后, 籽粒 GBSS 活性与对照差异较小; 高温解除后, 籽粒 GBSS 活性均低 于对照, 但与对照差异较小。可见, 与其他籽粒淀粉合成相 关酶相比，高温对籽粒 GBSS 活性影响程度较小。

3 讨论

多数研究表明, 小麦花后高温显著降低籽粒淀粉及 支链淀粉含量 ${ }^{[6,9-10]}$ 。本研究亦表明同样结果, 且不同品种 对高温的反应不同。直链淀粉含量是影响面食品质的重要 因素之一，其含量的微弱变化即可导致面食加工品质的 明显不同 ${ }^{[16]}$ 。而前人关于高温对籽粒直链淀粉含量影响 的研究结果并不一致。Stoddard ${ }^{[17]}$ 研究认为, 在高温条件 下, 一些品种的直链淀粉含量没有明显改变, 另一些品种 的直链淀粉含量略有提高。赵辉等 ${ }^{[18]}$ 研究认为高温使直 链淀粉含量下降。本研究表明, 灌浆期高温提高籽粒直链 淀粉含量, 进而显著提高直/支比。可见, 灌浆期高温改变 了成熟期籽粒淀粉组分的构成比例。

淀粉粒级分布是淀粉品质的重要决定因素，不同大 小的淀粉粒对淀粉特性有显著影响。小麦籽粒不同粒级淀 粉粒分布除受基因型影响外，还受生态环境条件的影响。 Blumenthal等 ${ }^{[7]}$ 研究认为, 灌浆期高温提高籽粒 $\mathrm{A}$ 型淀粉 粒的数目, 减少B型淀粉粒的数目。淀粉粒的形成是一个 受生长发育调节的过程，灌浆期不同阶段高温对淀粉粒 的形成产生不同的影响。但前人关于灌浆前期高温对淀粉 粒度分布的研究较少。本研究结果表明, 灌浆前期高温不 仅影响籽粒 $\mathrm{A} 、 \mathrm{~B}$ 型淀粉粒的数目，而且对淀粉粒的体积、 表面积亦有显著影响。即高温显著增加籽粒 $\mathrm{A}$ 型淀粉粒的 体积、数目和表面积百分比，降低B型淀粉粒的体积、数 目和表面积百分比。原因可能是 $\mathrm{A}$ 型淀粉粒形成较早，且 小淀粉粒是由大淀粉粒分化而来 ${ }^{[19]}$ 。因此灌浆前期高温 降低籽粒小淀粉粒比例提高大淀粉粒比例。

研究表明, $\mathrm{A}$ 型淀粉粒的直链淀粉含量较高, 约为 $30 \%$ 36\%，B型淀粉粒的直链淀粉含量较低，约为 $24 \% \sim 27 \%{ }^{[20]}$ 。由本研究可知, 高温显著提高籽粒 $\mathrm{A}$ 型淀粉 粒比例, 这也与高温提高籽粒直链淀粉含量、直/支链淀粉 比的结果一致。

前人对灌浆期高温条件下，小麦籽粒蔗糖含量和淀 粉合成之间的关系有已有较多的研究 ${ }^{[6,9-10]}$ 。Jenner等 ${ }^{[9]}$ 研 究认为, 高温环境下小麦籽粒淀粉含量的下降, 并不是由 于光合产物的供给 ${ }^{6]}$ 不足。本研究表明, 灌浆前期高温使 籽粒蔗糖含量于花后 15 20 d后开始显著低于对照。结合 高温对籽粒SS活性的影响(图 2)可以看出，高温抑制了淀 粉合成原料蔗糖向籽粒的供应。

Jenner等 ${ }^{[9]}$ 研究表明, 灌浆期高温主要通过抑制SSS 活性, 阻遏蔗糖向淀粉的转化, 进而降低淀粉含量和粒 重。在本研究中, 两小麦品种处理间籽粒AGPP、SSS和 
GBSS活性变化趋势, 与其籽粒淀粉积累变化趋势基本一 致。可见灌浆期高温处理中淀粉积累量下降与其籽粒 AGPP、SSS、GBSS活性下降显著相关。GBSS是直链淀 粉合成的关键酶, SSS 是支链淀粉合成的关键酶 ${ }^{[15,21]}$ 。本 研究发现, 与籽粒AGPP、SSS活性相比, 灌浆期高温对 GBSS活性的影响相对较小。可见与直链淀粉相比，小麦 籽粒支链淀粉的合成更易受到高温抑制, 这可能也是本 试验条件下小麦直链淀粉含量相对升高的原因之一。小麦 灌浆期高温使籽粒淀粉积累量降低, 主要因高温抑制了 籽粒灌浆中后期的淀粉合成, 这是由籽粒蔗糖供应不足 和籽粒淀粉合成相关酶活性下降所造成的。

\section{References}

[1] Yao D-N (姚大年), Li B-Y (李保云), Zhu J-B (朱金宝), Liang R-Q (梁荣奇), Liu G-T (刘广田). Study on main starch properties and predictive indexes of noodle quality in common wheat (Triticum aestivum). Sci Agric Sin (中国农业科学), 1999, 32(6): 84-88 (in Chinese with English abstract)

[2] Parker M L. The relationship between A-type and B-type starch granules in the developing endosperm of wheat. J Cereal Sci, 1985, 3: 271-278

[3] Tang H, Ando H, Watanade K. Some physiological properties of small, medium and large granule starches in fractions of waxy barley grain. Cereal Chem, 2000, 77: 27-31

[4] Peng M, Gao M. Separation and characterization of A- and B-type starch granules in wheat endosperm. Cereal Chem, 1999, 76: 375-379

[5] Bechtel D B, Zayas I, Kaleikau L, Pomeranz Y. Size-distribution of wheat starch granules during endosperm development. Cereal Chem, 1990, 67: 59-63

[6] Sofield I, Evans L T, Cook M G, Wardlaw I F. Factors influencing the rate and duration of grain filling in wheat. Aust J Plant Physiol, 1977, 4: 785-797

[7] Blumenthal C, Bekes F, Gras P W, Barlow E W R, Wrigley C W. Identification of wheat genotypes tolerant to the effects of heat stress on grain quality. Cereal Chem, 1995, 72: 539-544

[8] Xu R-Q (徐如强), Sun Q-X (孙其信), Zhang S-Z (张树榛). Current status and prospective on the investigation of heat tolerance in wheat. J China Agric Univ (中国农业大学学报), 1998, 3(3): 33-40 (in Chinese with English abstract)

[9] Jenner C F. Effects of exposure of wheat ears to high temperature on dry matter accumulation and carbohydratemetabolism in the grain of two cultivars: I. Immediate responses. Aust J Plant Physiol, 1991, 18:
165-177

[10] Keeling P L, Banisadr R, Barone L, Wasserman B P, Singleary G W. Effects of temperature on enzymes in the pathway of starch biosynthesis in developing wheat and maize grain. Aust J Plant Physiol, 1994, 21: 807-827

[11] AI-Khatib K, Paulsen G M. Photosynthesis and productivity during high temperature stress of wheat genotypes from major world regions. Crop Sci, 1990, 30: 1127-1132

[12] Xu X L, Wang Z M, Zhang J P. Effects of heat stress on photosynthetic characteristics of different green organs of winter wheat during grain filling stage. Acta Bot Sin, 2001, 43: 571-577

[13] He Z-F (何照范). Analysis Technique for Grain Quality in Cereals and Oils (粮油籽粒品质及其分析技术). Beijing: Agriculture Press, 1985. pp 144-294 (in Chinese)

[14] Douglas C D, Kuo T M, Felker F C. Enzymes of sucrose and hexose metabolism in developing kernels of two inbreds of maize. Plant Physiol, 1988, 86: 1013-1019

[15] Nakamura Y, Yuki K, Park S Y. Carbohydrate metabolism in the developing endosperm of rice grains. Plant Cell Physiol, 1989, 56: 833-839

[16] Zeng M, Morris C F, Batey I L, Wrigley C W. Sources of variation for starch gelatinization, pasting, and gelation propertiesof wheat. Cereal Chem, 1997, 74: 63-71

[17] Stoddard F L. Survey of starch particle-size distribution in wheat and related species. Cereal Chem, 1999, 76: 145-149

[18] Zhao H (赵辉), Dai T-B (戴廷波), Jing Q (荆奇), Jiang D (姜东), Cao W-X (曹卫星), Lu W (陆玮), Tian X-W (田孝威). Effects of high temperature during grain filling on key enzymes involved in starch synthesis in two wheat cultivars with different quality types. Acta Agron Sin (作物学报), 2006, 32(3): 423-429 (in Chinese with English abstract)

[19] Sheng J (盛婧), Guo W-S (郭文善), Zhu X-K (朱新开), Feng C-N (封超年), Peng Y-X (彭永欣). Starch granules development in grain endosperm of wheat for different end uses. Acta Agron Sin (作物学 报), 2004, 30(9): 953-954 (in Chinese with English abstract)

[20] Hurkman W J, McCue K F, Altenbach S B. Effect of temperature on expression of genes encoding enzymes for starch biosynthesis in developing wheat endosperm. Plant Sci, 2003, 164: 873-881

[21] Yan S-H (间素辉), Wang Z-L (王振林), Dai Z-M (戴忠民), Li W-Y (李 文阳), Fu G-Z (付国占), He M-R (贺明荣), Yin Y-P (尹燕枰). Activities of enzymes involved in starch synthesis and accumulation in grains of two wheat cultivars with a different amylose content. Acta Agron Sin (作物学报), 2007, 33(1): 84-89 (in Chinese with English abstract) 